\title{
BINGO QUÍMICO: UMA ATIVIDADE LÚDICA ENVOLVENDO FÓRMULAS E NOMENCLATURAS DOS COMPOSTOS
}

\author{
F. B. F. Moreira, M. V. de O. Costa $^{1}$, E. M. Barbosa ${ }^{1}$ e L. M. Bertini ${ }^{1}$ \\ Instituto Federal de Educação, Ciência e Tecnologia do Rio Grande do Norte - Câmpus Apodi \\ belkisemoreira@hotmail.com - m_vini_doc@hotmail.com - eryv @rocketmail.com - luciana.bertini@ifrn.edu.br
}

Artigo submetido em setembro/2012 e aceito em dezembro/2012

\section{RESUMO}

O jogo visa estimular o raciocínio lógico, proporcionando uma forma dinâmica de aprendizado e construção de conhecimentos. Desta forma, reconhecendo a relevância deste recurso tão importante, este trabalho tem como objetivo desenvolver um jogo, intitulado "Bingo Químico", para auxiliar o ensino das fórmulas e nomenclatura dos compostos. Antes da aplicação do jogo foi ministrada uma aula teórica sobre o assunto em questão e logo após foi realizado um teste de sondagem para avaliar o nível da aprendizagem. Em seguida, o jogo foi aplicado com 37 alunos da 2a série do ensino médio técnico integrado em Biocombustível do IFRN campus Apodi. Após a aplicação do jogo, foi aplicado um teste de sondagem e um levantamento de dados para saber como foi à aceitação do jogo. A dinâmica ocorreu como um bingo tradicional, porém não foram sorteados números, e sim os nomes dos compostos. Os resultados obtidos pela análise dos dados coletados do teste de sondagem após o jogo foram satisfatórios. O percentual de notas abaixo e acima de 6,0 foram respectivamente $32 \%$ e $68 \%$, tendo uma melhora de $14 \%$ com relação ao teste de sondagem antes do jogo. O jogo Bingo Químico mostrou ser um recurso lúdico válido, tornando o ensino de Química no ensino médio mais contextualizado, interessante e divertido.

PALAVRAS-CHAVE: Bingo Químico, jogos educativos, estrutura e nomenclatura dos compostos químicos.

\section{CHEMICAL BINGO: A PLAYFUL ACTIVITY INVOLVING FORMULAS AND NOMENCLATURES OF COMPOSITES}

\section{ABSTRACT}

The game aims to stimulate the logical reasoning, providing a dynamic way of learning and knowledge construction. This way, recognizing the relevance of this most important resource, this work aims to develop a game, entitled "Chemical Bingo", to assist the teaching of the formulas and nomenclature of compounds. Before the implementation of the game was given a theoretical lesson on the subject in question and soon after was conducted a test of survey to evaluate the level of learning. Then, the game was applied with 37 students of the 20 series of middle school integrated technical in Biofuel of IFRN campus Apodi. After the application of the game, was applied a test poll and a survey of data to know how was the acceptance of the game. The dynamic occurred as a traditional bingo, but were not randomly selected numbers, and yes the names of the compounds. The results obtained by the analysis of data collected from test poll after the game was satisfactory. The percentage of notes below and above 6.0 was respectively $32 \%$ and $68 \%$, an improvement of $14 \%$ in relation to the test poll before the game. The game Bingo Chemical proved to be a playful appeal valid, making the teaching of Chemistry in high school more contextualized, interesting and fun.

KEY WORDS: Chemical bingo, educational games, structure and nomenclature of chemical compounds. 


\section{BINGO QUÍMICO: UMA ATIVIDADE LÚDICA ENVOLVENDO FÓRMULAS E NOMENCLATURAS DOS COMPOSTOS}

\section{INTRODUÇÃO}

O ensino da Química é de fundamental importância na formação da cidadania, pois esta ciência faz parte da sociedade tecnológica moderna. As ciências têm sido ensinadas como uma coleção de fatos, descrição de fenômenos, enunciados de teorias em que o aluno tem que memorizar (SANTOS, 2004). A maioria dos educadores não procura fazer com que os alunos discutam as causas dos fenômenos, estabeleçam relações da ciência com o cotidiano, enfim, que entendam os mecanismos dos processos que estão estudando (ZANON, 2008).

Segundo Silva et al (2009) é muito comum que não seja dada a devida importância ao que é chamado, na literatura, de processo da ciência, ou seja, aos eventos e procedimentos que levam às descobertas científicas. Em geral, o ensino fica limitado à apresentação dos chamados produtos da ciência. Assim, como afirma Domingos e Recena (2010), para muitos alunos, aprender Química é decorar um conjunto de nomes, fórmulas, descrições de instrumentos ou substâncias, enunciados de leis.

Diante dessas circunstâncias, as atividades lúdicas, mais do que aceitas como rotina da educação dos alunos, são uma prática privilegiada para a aplicação de uma educação que visa o desenvolvimento pessoal e a atuação cooperativa na sociedade, como também instrumentos motivadores, atraentes e estimuladores do processo de construção do conhecimento, como bem define Soares (2004) ao considerá-las como uma ação divertida, seja qual for o contexto linguístico, desconsiderando o objeto envolto na ação.

O jogo, considerado como um tipo de atividade lúdica, segundo Kishimoto (1996 apud SANTANA, 2006) possui duas funções: a lúdica e a educativa, em que as mesmas devem coexistir em equilíbrio, sem que nenhuma prevaleça sobre a outra. Segundo Cunha (1998) o lúdico apresenta dois elementos que o caracterizam: o prazer e o esforço espontâneo, além de integrarem as várias dimensões do aluno, como a afetividade, o trabalho em grupo e das relações com regras pré-definidas. Segundo Borges e Schwarz (2005) com as atividades lúdicas o indivíduo torna-se capaz de mobilizar de forma criativa e eficaz as suas habilidades, nas quais os conhecimentos, valores e atitudes são usados de forma integrada frente às necessidades impostas pelo meio.

Desta forma, o presente trabalho tem como finalidade mostrar o desenvolvimento de um jogo lúdico, intitulado "Bingo Químico", para auxiliar o ensino das fórmulas e nomenclatura dos compostos.

\section{METODOLOGIA}

O processo de realização deste trabalho foi dividido em etapas. A primeira foi à confecção do jogo. Foram selecionados 60 compostos químicos para serem utilizados no bingo, possuindo em cada cartela 30 compostos de forma aleatória. Nas cartelas continham apenas as fórmulas dos compostos como é mostrado na Figura 1. Os materiais utilizados na construção das cartelas foram: cartolina guache preta para servir de base para as cartelas do 
bingo, papel ofício, fita transparente para fixar as cartelas em sua respectiva base, tesoura, computador e impressora.

\begin{tabular}{|c|c|c|c|c|}
\hline $\begin{array}{c}\text { Bingo } \\
(\mathrm{B})\end{array}$ & $\begin{array}{c}\text { Químico } \\
(\mathrm{Q})\end{array}$ & $\begin{array}{c}\text { Bingo } \\
(\mathrm{B})\end{array}$ & $\begin{array}{c}\text { Químico } \\
(\mathrm{Q})\end{array}$ & $\begin{array}{c}\text { Bingo } \\
(\mathrm{B})\end{array}$ \\
\hline $\mathrm{HC}_{2} \mathrm{H}_{3} \mathrm{O}_{2}$ & $\mathrm{NiS}$ & $\mathrm{H}_{2} \mathrm{CO}_{3}$ & $\mathrm{MgC}_{2} \mathrm{O}_{4}$ & $\mathrm{H}_{5} \mathrm{IO}_{6}$ \\
\hline $\mathrm{H}_{2} \mathrm{SO}_{4}$ & $\mathrm{AgBrO}_{3}$ & $\mathrm{HClO}_{2}$ & $\mathrm{CoCO}_{3}$ & $\mathrm{HNO}_{2}$ \\
\hline $\mathrm{HC}_{7} \mathrm{H}_{5} \mathrm{O}_{2}$ & $\mathrm{PbF}_{2}$ & $\mathrm{H}_{3} \mathrm{AsO}_{4}$ & $\mathrm{NiCO}_{3}$ & $\mathrm{HF}$ \\
\hline $\mathrm{HCN}$ & $\mathrm{BaSO}_{4}$ & $\mathrm{HN}_{3}$ & $\mathrm{La}\left(\mathrm{IO}_{3}\right)_{3}$ & $\mathrm{HI}$ \\
\hline $\mathrm{H}_{2} \mathrm{~S}$ & $\mathrm{Ca}(\mathrm{OH})_{2}$ & $\mathrm{H}_{2} \mathrm{O}_{2}$ & $\mathrm{MnS}$ & $\mathrm{H}_{2} \mathrm{SO}_{3}$ \\
\hline $\mathrm{HC}_{6} \mathrm{H}_{5} \mathrm{O}$ & $\mathrm{Fe}(\mathrm{OH})_{2}$ & $\mathrm{HIO}$ & $\mathrm{BaF}_{2}$ & $\mathrm{H}_{3} \mathrm{PO}_{3}$ \\
\hline
\end{tabular}

\section{Figura 1: Exemplo da Cartela do Bingo Químico}

Tornou-se necessário a confeç̧ão de 60 peças dos compostos químicos para serem sorteadas no bingo. Nessas 60 peças que foram utilizadas no sorteio continham as fórmulas, nomes dos compostos e a letra onde o composto se encontrava como é mostrado na Figura 2. Os materiais utilizados para a confecção dessas peças foram cartolina guache verde, tesoura, papel ofício, fita transparente e uma caixa para guardar as peças.

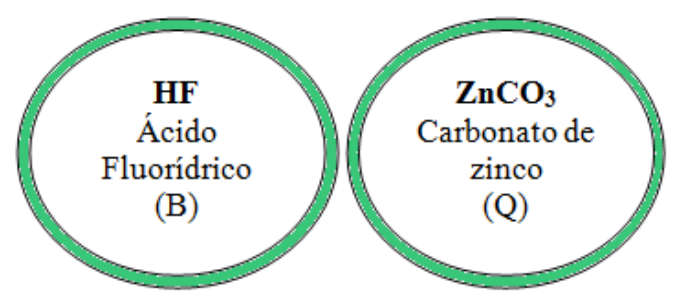

Figura 2: Exemplo das peças do Bingo Químico

Inicialmente os alunos tiveram uma aula sobre a nomenclatura dos compostos químicos inorgânicos e em seguida foi aplicado um teste de sondagem para avaliar o nível de aprendizagem.

Um segundo momento ocorreu com a apresentação do jogo onde foram expostas as regras e estratégias que eram idênticas a um bingo tradicional, porém o reconhecimento da nomenclatura e a fórmula química eram primordiais para que o jogador pudesse marcar a sua cartela, o que obrigava o aluno/jogador a identificá-las corretamente. Nesta etapa da atividade dividiu-se os 37 alunos em grupos de 2 ou 3, permitindo aos mesmos, num primeiro momento, a familiarização com o seu material e de como seriam as regras e a prática do jogo.

Para dar início ao jogo foi distribuída uma cartela para cada grupo e grãos de milho para marcação do composto na cartela. Foi necessária a interrupção do jogo durante alguns minutos, em algumas equipes, para fazer um levantamento das dificuldades e dúvidas sobre o assunto explicado. À medida que foram sendo chamadas as peças os alunos marcavam nas suas cartelas os compostos sorteados e após alguns segundos os mesmos eram escritos no quadro para que os alunos pudessem verificar se estavam corretos. $O$ jogo só acabava quando toda uma linha ou coluna fossem totalmente preenchidas. Nesse momento, a equipe deveria 
gritar a palavra: Químico ao invés de Bingo. Assim, o sorteio era interrompido e o professor e os bolsistas conferiam a cartela. Em seguida, o jogo continuava até que tivessem três grupos vencedores. No jogo havia uma premiação para os grupos que ganhassem em primeiro, segundo e terceiro lugar.

Após a aplicação do jogo foi realizado um segundo teste de sondagem para a verificação da aprendizagem. A última etapa foi caracterizada pela entrega de fichas de avaliação aos alunos contendo afirmações com respeito à atividade desenvolvida.

\section{RESULTADOS E DISCUSSÃO}

A aplicação do Bingo Químico envolveu 37 alunos da turma da 2a série do Ensino Médio Técnico Integrado em Biocombustíveis do IFRN/Apodi, os quais mostraram um grande interesse pelo jogo e estavam felizes por participarem ativamente da atividade.

No teste de sondagem feito antes do jogo pôde-se perceber e comprovar com os resultados obtidos, que os alunos tiveram dificuldades para responder algumas das questões, principalmente quando se tratava de escrever a nomenclatura de determinados compostos, resultando em algumas questões em branco.

O teste continha seis questões envolvendo fórmulas e nomes de compostos químicos. A cada questão foi atribuída uma nota, somando-se dez pontos no total. Tiveram notas elevadas, porém muitas abaixo da média. A Tabela 1 mostra o percentual das notas obtidas antes do jogo.

Tabela 1: Resultados do teste de sondagem antes e após o jogo.

\begin{tabular}{|c|c|c|}
\hline Notas & $\begin{array}{c}\text { Percentagem } \\
\text { antes do jogo (\%) }\end{array}$ & $\begin{array}{c}\text { Percentagem } \\
\text { após o jogo (\%) }\end{array}$ \\
\hline Abaixo de 6,0 & 46 & 32 \\
\hline Acima de 6,0 & 54 & 68 \\
\hline
\end{tabular}

Observando a Tabela 1, percebeu-se que $54 \%$ dos alunos obtiveram uma nota acima de 6,0 enquanto que $46 \%$ as notas foram inferiores a média escolar. Talvez por se tratar de um assunto que requer muita prática, uma parte dos alunos não obteve êxito, e o percentual abaixo da média foi bastante preocupante.

Durante a aplicação do jogo pôde-se observar a curiosidade dos alunos pelo assunto trabalhado, a socialização entre os grupos, a interação da turma, a diversão e ao mesmo tempo uma aula mais dinâmica, tornado mais significativa à aprendizagem como é observado na Figura 4. 

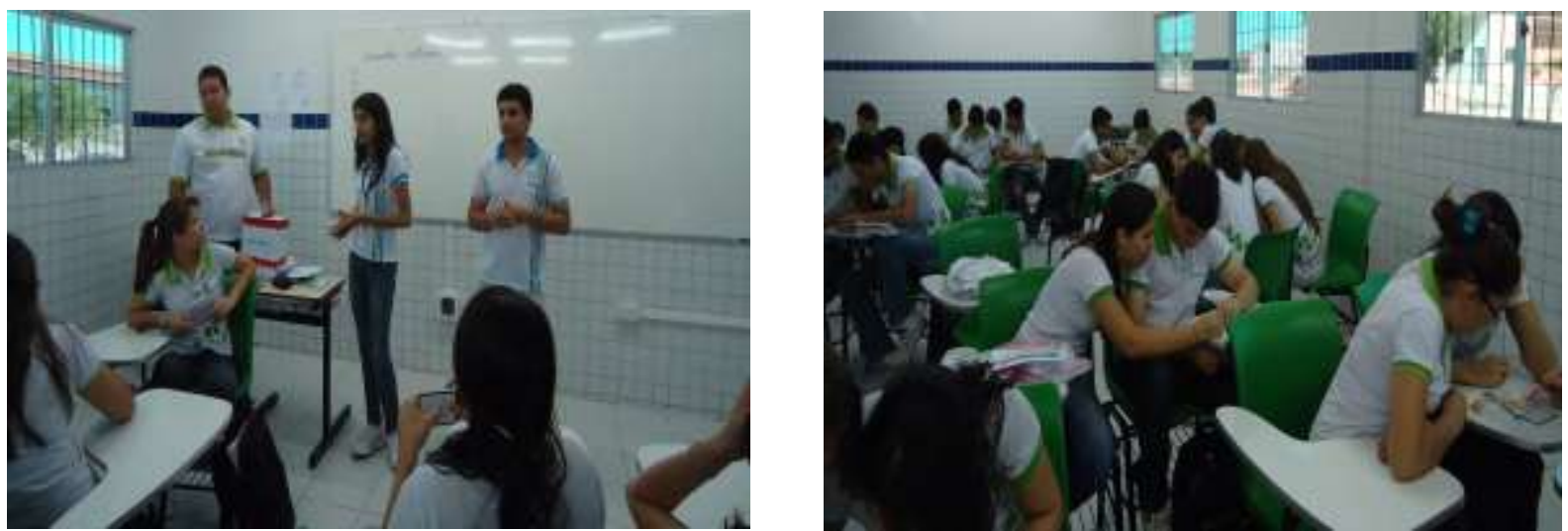

Figura 4: Explicação e aplicação do Bingo Químico

Após a aplicação do jogo foi feito outro teste de sondagem. Ao comparar os resultados obtidos nesse teste com o primeiro pôde-se perceber que o jogo ajudou a melhorar o desempenho dos alunos com relação ao conhecimento sobre a nomenclatura dos compostos químicos como pode ser observado na Tabela 1.

De acordo com a Tabela 1, o jogo foi uma ferramenta lúdica bastante importante que contribuiu para essa melhora, pois é possível verificar que os alunos que possuíam dificuldades com a matéria ministrada, após jogarem o Bingo Químico as mesmas foram minimizadas, efetivando assim o processo de aprendizagem e o interesse pela Química.

Para analisar o nível de aceitação do jogo na sala, os alunos responderam a um questionário respondendo sim ou não para as perguntas. Os questionamentos estão contidos na Tabela 2 abaixo.

Tabela 2: Perguntas avaliativas do Bingo Químico.

\begin{tabular}{|l|}
\hline \multicolumn{1}{|c|}{ Perguntas } \\
\hline 1- Os jogos auxiliam na fixação do conteúdo? \\
\hline 2- Aprende-se melhor um conteúdo, introduzindo-o com jogos? \\
\hline $\begin{array}{l}\text { 3- Ajuda a melhorar os relacionamentos por ser uma atividade } \\
\text { desenvolvida em grupo? }\end{array}$ \\
\hline 4- Auxilia para que a aula se torne mais atrativa? \\
\hline 5- A metodologia é melhor do que uma aula expositiva? \\
\hline $\begin{array}{l}\text { 6- Despois do jogo aumentou seu interesse em estudar mais o } \\
\text { conteúdo da disciplina? }\end{array}$ \\
\hline 7- O jogo aplicado é de fácil compreensão? \\
\hline $\begin{array}{l}\text { 8- Trabalhando o conteúdo em grupo o jogo foi possível sanar } \\
\text { algumas dificuldades? }\end{array}$ \\
\hline 9- Pode-se dividir a aula em: expositiva, exercícios e jogo? \\
\hline $\begin{array}{l}\text { 10- Atividades com jogos podem ser trabalhados em outras } \\
\text { disciplinas? }\end{array}$ \\
\hline 11- Aprovei esse tipo de atividade? \\
\hline
\end{tabular}

Os resultados dos questionamentos que se refere na Tabela 2 estão contidos na Figura 5 abaixo. 


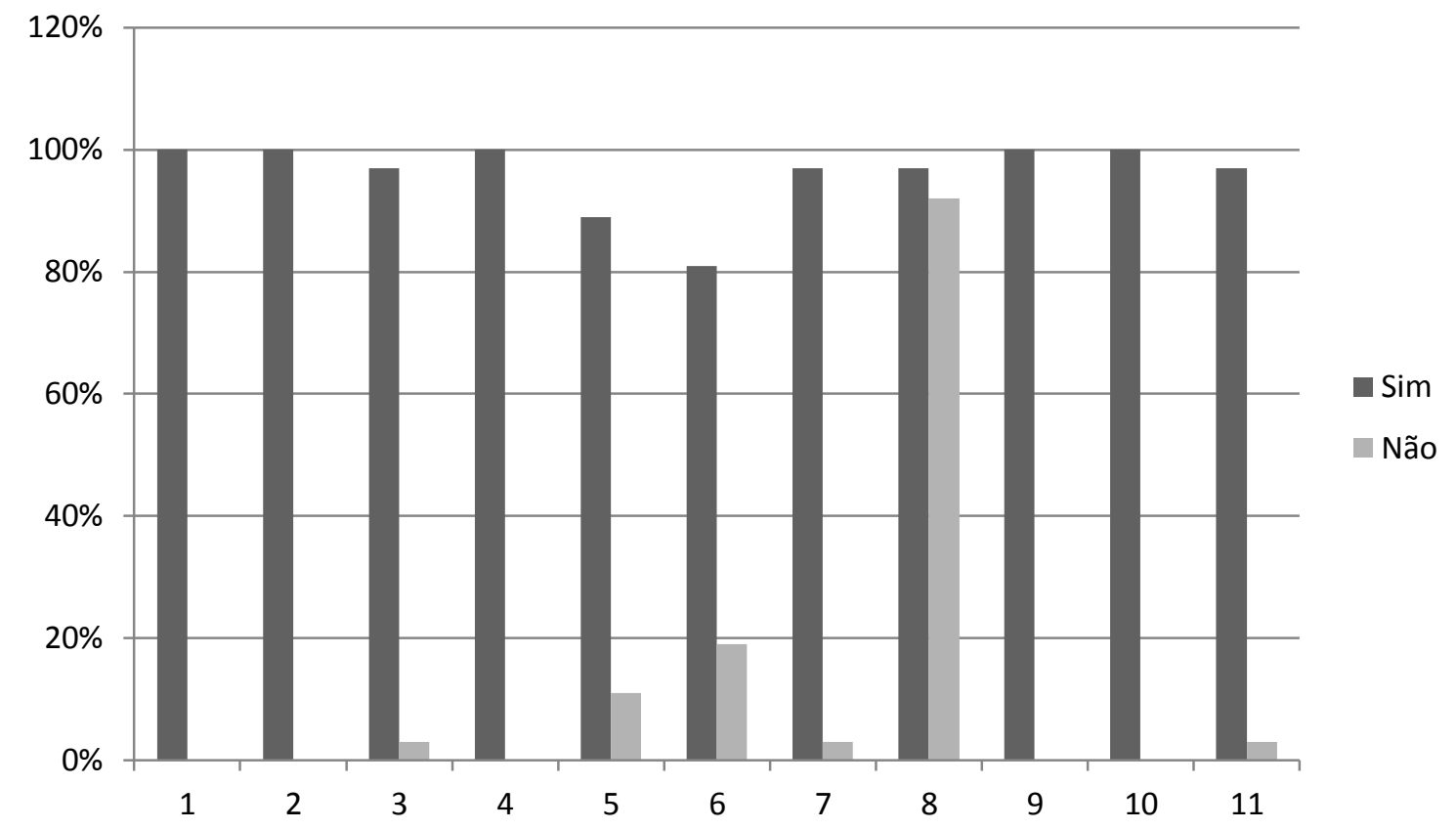

Figura 5: Avaliação do Bingo Químico

Diante desses resultados pôde-se perceber que o jogo foi bem aceito pelos alunos, pois ao complementar a aula teórica com exercícios e com jogos lúdicos, a mesma se tornou mais atrativa, divertida e interessante.

A importância da utilização de atividades lúdicas mobilizam esquemas mentais, ativando funções neurológicas e psicológicas estimulando o pensamento. E quando o sujeito está diretamente envolvido na ação fica mais fácil à compreensão do aspecto cognitivo, pois ocorre um desbloqueio mental (SANTANA, 2006).

Os jogos são um importante recurso não só para as aulas de Química, mas como para qualquer outra disciplina, pois facilitam a aprendizagem do aluno, além de motivar o mesmo. Podendo ser trabalhadas diretamente as habilidades dos alunos além de existir uma maior socialização entre os colegas da turma, entre o aluno e o professor, entre o aluno e o jogo e entre o mesmo e o conteúdo a ser trabalhado.

\section{CONSIDERAÇÕES FINAIS}

O desenvolvimento de jogos corretamente criados e devidamente escolhidos com seus objetivos definidos proporciona aos alunos diversas interações, promovendo construções e aperfeiçoamentos de conceitos, habilidades e a valorização do conhecimento, resgatando as lacunas que o processo de ensino e aprendizagem atual deixa em aberto. A função educativa do jogo "Bingo Químico" foi facilmente observada durante sua aplicação ao verificar o favorecimento da aquisição de conhecimento em clima de alegria e prazer. Cabe ressaltar que os jogos pedagógicos não são substitutos de outros métodos de ensino, eles são apenas suportes para o professor e poderosos motivadores para os alunos que usufruírem os mesmos, como recurso didático para a sua aprendizagem. 


\section{AGRADECIMENTOS}

Os autores agradecem ao Instituto Federal de Educação, Ciência e Tecnologia do Rio Grande do Norte pelo fornecimento das bolsas de pesquisa na modalidade PIBIC-IFRN e a participação dos alunos da $2^{a}$ série do ensino médio técnico integrado em Biocombustível do IFRN campus Apodi.

\section{REFERÊNCIAS BIBLIOGRÁFICAS}

1. BORGES, R. M. R.; SCHWARZ, V. O. O Papel dos jogos educativos no processo de qualificação de professores de ciências. In: IV Encontro Ibero-Americano coletivos escolares e redes de professores que fazem investigação na escola, 4. Lajeado. ed. UNIVATES, 2005.

2. CUNHA, H.S. Brinquedo, desafio e descoberta. 1ạ edição. AE/MEC/RJ, 1998.

3. DOMINGOS, D. C. A.; RECENA, M. C. P. Elaboração de jogos didáticos no processo de ensino e aprendizagem de química: a construção do conhecimento. Ciências \& Cognição (UFRJ), Rio de Janeiro, v. 15, 2010.

4. KISHIMOTO, T.M. Jogo, Brinquedo, Brincadeira e a Educação. São Paulo: Cortez, 1996.

5. SANTANA, E. M. A Influência de atividades lúdicas na aprendizagem de conceitos químicos. Universidade de São Paulo, Instituto de Física - Programa de Pós-Graduação Interunidades em Ensino de Ciências, 2006.

6. SANTOS, M. S. M. A. Roleta de Iões: uma nova aplicação para o ensino de Química. Dissertação de Mestrado, Programa de pós-graduação em Educação Multimídia, Universidade do Porto, 2004.

7. SILVA, L. M.; CRUZ, K. C. A.; FILHO, J. R. L.; REIS, S. M.; COSTA, P. H. C. S.; SOUZA, L.. Master Química: A utilização de jogos educativos como motivação para aprender Química. IFPB JP. Instituto Federal de Educação, Ciência e Tecnologia da Paraíba, 2009.

8. SOARES, M. H. F. B. O lúdico em Química: jogos e atividades aplicados ao ensino de Química. Tese (Doutorado em Ensino de Química), 2004. 71f. Universidade Federal de São Carlos, São Paulo. 2004.

9. ZANON, D. A. V.; GUERREIRO, M. A. S.; OLIVEIRA, R. C. Jogo didático Ludo Químico para o ensino de nomenclatura dos compostos orgânicos: projeto, produção, aplicação e avaliação. (UNESP)-Universidade Estadual Paulista Júlio de Mesquita Filho Campus Araraquara- São Paulo, 2008. 